\title{
TRAJETÓRIA CRONOLÓGICA DO PROFISSIONAL DE EDUCAÇÃO FÍSICA
}

\author{
Cassio Hartmann ${ }^{1}$ \\ Gabriel César Dias Lopes² \\ Fábio da Silva Ferreira Vieira ${ }^{3}$ \\ Bensson V Samuel ${ }^{4}$
}

\section{RESUMO}

O presente estudo teve como objetivo, apresentar a trajetória dos profissionais de Educação Física, desde a década de 1977 até o período de 2020, aonde a categoria conquista permanentemente através da CBO Classificação Brasileira de Ocupações, a inserção do profissional de Educação Física na Saúde, que ocorreu em 17 de fevereiro de 2020 com o código 2241-40. A referida pesquisa caracteriza-se como documental parecida com a pesquisa bibliográfica. Diante das considerações finais dessa pesquisa, o profissional de Educação Física, deverá e conseguirá desenvolver ações que propiciem a melhoria da qualidade de vida da população, a fim de reduzir os agravos e danos decorrentes das doenças não-transmissíveis, que favoreçam a redução do consumo de medicamentos e principalmente trabalhar em equipe multidisciplinar do SUS Sistema Único de Saúde.

Palavras-chaves: Cronológica, Classificação Brasileira de Ocupações, Educação Física.

\footnotetext{
${ }^{1}$ Professor de Educação Física SEM FRONTEIRAS DA FIEP-BRASIL / Delegado Adjunto Nacional da Federação Internacional de Educação Física FIEP / Secretario e Imortal da ABEF - Academia Brasileira de Educação Física / Conselheiro CREF 19AL / Professor de Educação Física do Instituto Federal de
} 
Alagoas/IFAL e doutorando em saúde coletiva com ênfase em Educação Física E-mail: cassiohartmann04@gmail.com

${ }^{2}$ Prof. Dr. Gabriel C. D. Lopes, PhD Professor e Orientador Doutor em Educação / PhD em Psicanálise Clínica Presidente da LUI - Logos University Int. Professor / Membro Imortal da ABEF - Academia Brasileira de Educação Física - E-mail: president@unilogos.education

${ }^{3}$ Professor Co-orientador / Delegado Adjunto da Federação Internacional de Educação Física FIEP-PR / Doutor em ciências do movimento humano. E-mail: vieira.fsf@gmail.com

${ }^{4}$ Professor de Ciências e Médico / Bacharel em Medicina Poznan University of Medical Science, Poland/ Bacharel em Ciências Médicas e Laboratoriais (Cito-Tecnologia) University of Connecticut, Storrs, CT, USA / Especialista em Clínica Geral Queen Mary University / Especialista em Urgência e Emergência Medvarsity-Apollo Hospital / Doutorado em Liderança e Gestão Estratégica London School of Internation Business / Doutorado PhD: Pan-American University - Health Care Management / Doutorado PhD: Swiss Open University in Economics. E-mail: besson123@yahoo.com

\section{ABSTRACT}

The present study aimed to present the trajectory of Physical Education professionals, from the 1977 to the 2020 period, where the category permanently conquers through the CBO Brazilian Classification of Occupations, the insertion of the Physical Education in Health professional, which occurred on February 17, 2020 under code 2241-40. This research is characterized as documentary, similar to bibliographic research. In view of the final considerations of this research, the Physical Education professional must and will be able to develop actions that promote the improvement of the population's quality of life, in order to reduce the injuries and damages resulting from non-communicable diseases, which favor the reduction of consumption medication and mainly working in a multidisciplinary team of SUS Unified Health System.

Keywords: Chronological, Brazilian Classification of Occupations, Physical Education.

\section{RESUMEN}

El presente estudio tenia como objetivo presentar la trayectoria de los profesionales de Educación Física, desde el período 1977 hasta el 2020, donde la categoría conquista permanentemente a través de la Clasificación de Ocupaciones de la CBO de Brasil, la inserción del profesional de Educación Física en Salud que ocurrió el 17 
de febrero de 2020 bajo el código 2241-40. Esta investigación se caracteriza por ser documental, similar a la investigación bibliográfica. En vista de las consideraciones finales de esta investigación, el profesional de Educación Física debe y podrá desarrollar acciones que promuevan la mejora de la calidad de vida de la población, a fin de reducir las agravaciones y daños resultantes de enfermedades no transmisibles, que favorecen la reducción del consumo medicamentos y principalmente trabajando en un equipo multidisciplinario del Sistema Único de Salud del SUS.

Palabras clave: Cronológica, Clasificación Brasileña de Ocupaciones, Educación Física.

\section{INTRODUÇÃO}

Desde o século $X X$ a área de Educação Física, vem ganhando destaque no mercado de trabalho e ao mesmo tempo vem preenchendo uma lacuna e vacuidade nos sistemas e órgãos governamentais de saúde e o Conselho Nacional de Saúde, vem reconhecer o profissional da Educação Física sendo da área de saúde, no 06 de março de 1997, através da Resolução no 218. De acordo com a Portaria 154/2008.

Diante do exposto faz-se necessário dissertar sobre a trajetória do profissional de Educação Física, todo o caminho percorrido desde a década de 1977 até o período de 2020.

A estrutura básica da CBO Classificação Brasileira de Ocupações, foi elaborado em 1977, resultado do convênio firmado entre o Brasil e a Organização das Nações Unidas - ONU, por intermédio da Organização Internacional do Trabalho OIT, no Projeto de Planejamento de Recursos Humanos (Projeto BRA/70/550), tendo como base a Classificação Internacional Uniforme de Ocupações - CIUO de 1968.

Coube a responsabilidade de elaboração e atualização da CBO ao MTE, com base legal nas Portarias no 3.654, de 24.11.1977, no 1.334, de 21.12.1994 e no 397 CBO 2002. É referência obrigatória dos registros administrativos que informam os diversos programas da política de trabalho do País. É ferramenta fundamental para as estatísticas de emprego-desemprego, para o estudo das taxas de natalidade e mortalidade das ocupações, para o planejamento das reconversões e requalificações 
ocupacionais, na elaboração de currículos, no planejamento da educação profissional, no rastreamento de vagas, dos serviços de intermediação de mão-de-obra.

Desde a sua publicação, a CBO sofreu atualizações pontuais, sem modificações estruturais e metodológicas. Entretanto, uma nova metodologia internacional foi publicada em 1988. Editada em espanhol CIUO 88, em inglês ISCO 88 e em francês CITP 88, sob os auspícios da OIT, a nova classificação alterou os critérios de agregação.

Esta pesquisa caracteriza-se, segundo Marconi \& Lakatos (2017) como qualitativa aquela que identifica fatores relevantes de um objeto de maneira a analisar o teor de documentos ou realidades, e ainda ressaltam a importância do embasamento teórico, assim sendo, o presente estudo parecido com a pesquisa bibliográfica se diferencia apenas pela natureza dos materiais pesquisados como fontes, esta está direcionada a um referencial que ainda não receberam um tratamento analítico, ou que ainda podem ser reelaborados de acordo com os objetos da pesquisa. Além de analisar os documentos primários (documentos de arquivos, igrejas, sindicatos, instituições, associações, etc.), podem também analisar documentos oriundos de interpretações, como relatórios empresariais, relatos de caso, entre outros, desde que sejam documentos validados.

O trabalho está dividido em introdução e mais cinco subtópicos. No primeiro esta descrito a história da CBO Classificação Brasileira de Ocupações, assim como a regulamentação da profissão de Educação Física e a sua trajetória na saúde. No segundo é discutido o conceito de ocupação, emprego ou situação de trabalho e os tipos de competências. No terceiro é citado a Portaria no 397 , de 09 de outubro de 2002 que aprova a Classificação Brasileira de Ocupações - CBO/2002, para uso em todo território nacional e em ordem cronológica o ano que o profissional de Educação Física é Incluído nas Equipes de Saúde da Família do Núcleo de Apoio à Saúde da Família (NASF), Criação do Código Provisório 2241-E1 pelo Ministério da Saúde, para o profissional de Educação Física. O Conselho Nacional de Saúde - CNS juntamente com a CBO torna o Código obrigatório para profissionais na Academia da Saúde e com a publicação da Lei 12.864, em 2013 inclui a atividade física como fator determinante e condicionante da saúde. No quarto a inclusão permanente na CBO com a classificação número 2241-40 - Profissional de Educação Física na Saúde. As considerações finais trazem a trajetória do profissional de Educação Física e a sua 
atuação nas ações de promoção da saúde mediante práticas corporais, atividades físicas e de lazer na prevenção primária, secundária e terciária no SUS Sistema único de Saúde e no setor privado.

\section{HISTÓRICO DA CBO, REGULAMENTAÇÃO DA PROFISSÃO DE EDUCAÇÃO FÍSICA E SUA TRAJETÓRIA NA SAÚDE.}

No Brasil, as informações administrativas relativas às ocupações eram codificadas seguindo a estrutura da CBO. Entretanto, os dados censitários e as pesquisas domiciliares seguiam uma outra nomenclatura. $O$ Instituto Brasileiro de Geografia e Estatística - IBGE possuía uma nomenclatura própria, sem descrições.

A multiplicidade de classificações ocupacionais usadas no Brasil dificultava a comparabilidade entre os usuários de diferentes fontes de informações produzidas no território nacional, com o agravante de dificultar a comparação dessas estatísticas com aquelas geradas em outros países. $O$ trabalho inicial constituiu-se no esforço de articulação entre os órgãos brasileiros que usavam diferentes classificações de ocupação, na tentativa de unificá-las.

Em 1994 foi instituída a Comissão Nacional de Classificações - Concla, organismo interministerial cujo papel é unificar as classificações usadas no território nacional. A partir daí iniciou-se um trabalho conjunto do Ministério do Trabalho MTE e o IBGE no sentido de construir uma classificação única.

Para facilitar a execução de um projeto de tal envergadura, a Divisão de Classificação Brasileira de Ocupações - DCBO decidiu modularizar a construção da nova classificação.

O primeiro módulo foi construído em trabalho cooperativo entre a Divisão da CBO do MTE e o Departamento de Emprego e Rendimento - Deren do IBGE que resultou na publicação, em 1996, da tábua de conversão que permitiu a comparação entre as estatísticas de ocupação que utilizavam a classificação IBGE 91 e os registros administrativos que utilizam a CBO 94, tais como a Relação Anual de Informações Sociais - Rais, Cadastro Geral de Empregados e Desempregados - Caged, Seguro Desemprego, e as estatísticas internacionais que usam a CIUO 68 e a CIUO 88 . A 
tábua de conversão compatibilizou apenas os títulos, sem contudo modificar os critérios de agregação dos grupos ocupacionais, bem como sem refazer sua definições.

O segundo módulo foi constituído pela elaboração e validação da estrutura, já com a alteração de conceitos de agregação, utilizando-se o modelo CIUO 88 com algumas adaptações. Este trabalho foi desenvolvido pelo MTE e o IBGE com apoio de consultoria contratada para este fim.

Em 1997, o Conselho Nacional de Saúde, reconhece o profissional da Educação Física sendo da área de saúde, no 06 de março, através da Resolução no 218. De acordo com a Portaria 154/2008.

No dia Em 1ํ de Setembro de 1998, o Presidente da República, Fernando Henrique Cardoso, sanciona a lei 9696/98, publicada no Diário Oficial da União em 02/09/98 que regulamenta a profissão de Educação Física e cria os respectivos Conselho Federal e Conselhos Regionais.

Após o profissional de Educação Física ser reconhecido pelo Conselho Nacional de Saúde e a Regulamentação de sua profissão a CBO de posse de uma estrutura como ponto de partida, inicia-se o terceiro módulo que incluiu a escolha de um modelo de descrição e a organização de uma rede de parceiros para a construção da classificação descritiva. Aonde adotou-se o método Dacum - Developing A Curriculum, adaptando-o para descrever famílias ocupacionais. A descrição-piloto foi feita pelo Senai, no Rio de Janeiro, em 1999, a primeira instituição parceira a ser conveniada. Em 2000 e 2001 foram treinados facilitadores de novos conveniados do MTE - Fipe - Universidade de São Paulo, Funcamp - Universidade de Campinas, Fundep - Universidade Federal de Minas Gerais. Os trabalhos foram concluídos em agosto de 2002.

Além das instituições conveniadas, o MTE contou com os serviços de uma consultoria nacional e com o treinamento dos facilitadores do método Dacum, feito por instituição canadense. Na fase de definição da nomenclatura, contou com a participação de uma perita da OIT.

A grande novidade do processo descritivo em relação à CBO anterior é que cada família ocupacional foi descrita por um grupo de 8 a 12 trabalhadores da área, em oficina de trabalho (ou painel) com duração de três dias, sendo dois dias de descrição e um dia de revisão, por outro comitê, também formado por trabalhadores. 
Ao todo, foram 1.800 reuniões-dia, em vários pontos do Brasil, com a participação de aproximadamente 7 mil trabalhadores.

A outra novidade foi a mudança de filosofia de trabalho na CBO, a partir do desenvolvimento de sua nova base - de uma publicação ocupacional que era atualizada pontualmente, em um corte no tempo, publicada em papel, passou-se a montagem de uma rede de informações organizada em banco de dados, apoiada por um conjunto de instituições conveniadas que atualiza a base de forma contínua e incrementa novos desenvolvimentos, sob a coordenação do MTE.

\section{BASES CONCEITUAIS}

Ocupação é um conceito sintético não natural, artificialmente construído pelos analistas ocupacionais. O que existe no mundo concreto são as atividades exercidas pelo cidadão em um emprego ou outro tipo de relação de trabalho (autônomo, por exemplo).

Ocupação é a agregação de empregos ou situações de trabalho similares quanto às atividades realizadas.

O título ocupacional, em uma classificação, surge da agregação de situações similares de emprego e/ou trabalho. Outros dois conceitos sustentam a construção da nomenclatura da CBO 2002:

Emprego ou situação de trabalho: definido como um conjunto de atividades desempenhadas por uma pessoa, com ou sem vínculo empregatício. Esta é a unidade estatística da CBO.

Competências mobilizadas para o desempenho das atividades do emprego ou trabalho.

O conceito de competência tem duas dimensões:

10 Nível de competência: é função da complexidade, amplitude e responsabilidade das atividades desenvolvidas no emprego ou outro tipo de relação de trabalho.

20 Domínio (ou especialização) da competência: relaciona-se às características do contexto do trabalho como área de conhecimento, função, atividade econômica, processo produtivo, equipamentos, bens produzidos que identificarão o tipo de profissão ou ocupação. 
A nova estrutura proposta agrega os empregos por habilidades cognitivas comuns exigidas no exercício de um campo de trabalho mais elástico, composto por um conjunto de empregos similares que vai se constituir em um campo profissional do domínio x, y e z.

A unidade de observação é o emprego, dentro de um conjunto de empregos mais amplo (campo profissional), onde o ocupante terá mais facilidade em se movimentar.

Assim, ao invés de se colocar a lupa de observação sobre os postos de trabalho, agregando-os por similaridades de tarefas, como era a tônica da CIUO 68 e CBO 82 e CBO 94, a CBO 2002 amplia o campo de observação, privilegiando a amplitude dos empregos e sua complexidade, campo este que será objeto da mobilidade dos trabalhadores, em detrimento do detalhe da tarefa do posto.

Estes conjuntos de empregos (campo profissional) são identificados por processos, funções ou ramos de atividades.

Para manter unidade de linguagem com a CIUO 88, estes campos profissionais são denominados de grupos de base ou família ocupacional. Esta é a unidade de classificação descritiva mais desagregada.

Assim como a ocupação, o grupo de base ou família ocupacional é uma categoria sintética, um construto, ou seja, ela é elaborada a partir de informações reais, mas ela não existe objetivamente. Analogamente, não existe um animal vertebrado, mas é possível classificar uma porção de animais reais que tenham vértebras, dentro dessa categoria ou construto.

Para oferecer ao usuário da classificação ocupacional uma ponte entre a realidade e a categoria sintética, é preservada a Estrutura Ampliada de Denominações, que é o Índice Analítico no qual o usuário localizará o código e o nome do grupo de base de um emprego tipo x, y ou z, com chave de conversão entre a estrutura da CBO 94 para a CBO 2002.

Nota: todas as ocupações que compunham a CBO 94 e que não foram excluídas, constam também das famílias ocupacionais (grupos de base) da CBO 2002. Porém, as ocupações constantes de uma determinada família ocupacional da CBO 94 podem estar distribuídas em diferentes famílias ocupacionais da CBO 2002, pois não há correspondência direta entre as famílias ocupacionais das duas estruturas (CBO 94 e CBO 2002). Assim, a comparabilidade entre as duas estruturas poderá se dar por meio de cinco dígitos da CBO 94 e seis dígitos da CBO 2002. 


\section{Portaria no 397, de 09 de outubro de 2002}

Aprova a Classificação Brasileira de Ocupações - CBO/2002, para uso em todo território nacional e autoriza a sua publicação.

O Ministro de Estado do Trabalho e Emprego, no uso da atribuição que lhe confere o inciso II do parágrafo único do art. 87 da Constituição Federal, resolve:

Art. 1- - Aprovar a Classificação Brasileira de Ocupações - CBO, versão 2002, para uso em todo o território nacional.

Art. 2 - Determinar que os títulos e códigos constantes na Classificação Brasileira de Ocupações - CBO/2002, sejam adotados;

I. nas atividades de registro, inscrição, colocação e outras desenvolvidas pelo Sistema Nacional de Emprego (SINE);

II. na Relação anual de Informações Sociais - (RAIS);

III. nas relações dos empregados admitidos e desligados - CAGED, de que trata a Lei № 4923, de 23 de dezembro de 1965;

IV. na autorização de trabalho para mão-de-obra estrangeira;

V. no preenchimento do comunicado de dispensa para requerimento do benefício Seguro Desemprego (CD);

VI. no preenchimento da Carteira de Trabalho e Previdência Social - CTPS no campo relativo ao contrato de trabalho;

VII. nas atividades e programas do Ministério do Trabalho e Emprego, quando for o caso;

Art. 30 - O Departamento de Emprego e Salário - DES da Secretaria de Políticas Públicas de Emprego deste Ministério baixará as normas necessárias à regulamentação da utilização da Classificação Brasileira de Ocupações (CBO).

Parágrafo único. Caberá à Coordenação de Identificação e Registro Profissional, por intermédio da Divisão da Classificação Brasileira de Ocupações, atualizar a Classificação Brasileira de Ocupações - CBO procedendo às revisões técnicas necessárias com base na experiência de seu uso.

Art. 4ำ - Os efeitos de uniformização pretendida pela Classificação Brasileira de Ocupações (CBO) são de ordem administrativa e não se estendem às relações de emprego, não havendo obrigações decorrentes da mudança da nomenclatura do cargo exercido pelo empregado. 
Art. 5ำ - Autorizar a publicação da Classificação Brasileira de Ocupação - CBO, determinando que o uso da nova nomenclatura nos documentos oficiais a que aludem os itens I, II, III e V, do artigo 2ํㅡㄹ será obrigatória a partir de janeiro de 2003.

Art. 6 - Fica revogada a Portaria no 1.334, de 21 de dezembro de 1994.

Art. $7^{\circ}$ - Esta Portaria entra em vigor na data de sua publicação.

Paulo Jobim Filho ministro de Estado do Trabalho e Emprego

Em 2008 o profissional de Educação Física é Incluído nas Equipes de Saúde da Família do Núcleo de Apoio à Saúde da Família (NASF).

Em 2013 - Criação do Código Provisório 2241-E1 pelo Ministério da Saúde, para o profissional de Educação Física.

Ainda em 2013 o Conselho Nacional de Saúde - CNS juntamente com a CBO torna o Código obrigatório para profissionais na Academia da Saúde.

Com Publicação da Lei 12.864, em 2013 inclui a atividade física como fator determinante e condicionante da saúde.

\section{INSERÇÃO DO PROFISSIONAL DE EDUCAÇÃO FÍSICA NA SAÚDE CÓDIGO 2241-40 DE 17 DE FEVEREIRO DE 2020}

A Classificação Brasileira de Ocupações - CBO, do Ministério do Trabalho passou a apresentar um novo código: 2241-40, Profissional de Educação Física na Saúde. A publicação foi feita no dia 17 de fevereiro de 2020. Com a inclusão permanente, específica para atuação na Saúde, o profissional de Educação Física passa a integrar, de forma mais clara e objetiva as equipes dos Programas de Atenção Básica do SUS, bem como, possibilita a inclusão na Tabela de Prestação de Serviços do SUS.

O Profissional de Educação Física já fazia parte, desde 2002, com outras classificações relacionadas a atividades físicas e esportivas, a saber: 2241-05 Avaliador físico; 2241-10 - Ludomotricista; 2241-15 - Preparador de atleta; 2241-20 - Preparador físico; 2241-25 - Técnico de desporto individual e coletivo (exceto futebol); 2241-30 - Técnico de laboratório e fiscalização desportiva; 2241-35 Treinador profissional de futebol.

Com a inclusão da classificação número 2241-40 - Profissional de Educação Física na Saúde, a descrição primária foi ampliada com a seguinte informação: 
Coordenam, desenvolvem e orientam, com crianças, jovens e adultos, atividades físicas e práticas corporais. Ensinam técnicas desportivas; realizam treinamentos especializados com atletas de diferentes esportes; instruem-Ihes acerca dos princípios e regras inerentes a cada um deles; avaliam e supervisionam o preparo físico dos atletas; acompanham e supervisionam as práticas desportivas. Estruturam e realizam ações de promoção da saúde mediante práticas corporais, atividades físicas e de lazer na prevenção primária, secundária e terciária no SUS e no setor privado.

Importante ressaltar, que nas características do trabalho consta que "o exercício das ocupações da família requer formação superior em Educação Física, com registro no Conselho Regional de Educação Física".

\section{CONSIDERAÇÕES FINAIS}

Ao longo da história a trajetória do profissional de Educação Física, segue uma linha cronológica passando sua história inicial pelo Conselho Nacional de Saúde, que reconhece o profissional da Educação Física sendo da área de saúde, desde 06 de março de 1997, através da Resolução no 218. De acordo com a Portaria 154/2008, desde a elaboração da CBO Classificação Brasileira de Ocupações em 1977, até a sua aprovação, Portaria n 397, de 09 de outubro de 2002 para uso em todo território nacional.

Em 2008 o profissional de Educação Física é Incluído nas Equipes de Saúde da Família do Núcleo de Apoio à Saúde da Família (NASF).

Em 2013 - Criação do Código Provisório 2241-E1 pelo Ministério da Saúde, para o profissional de Educação Física.

Ainda em 2013 o Conselho Nacional de Saúde - CNS juntamente com a CBO torna o Código obrigatório para profissionais na Academia da Saúde e com Publicação da Lei 12.864, em 2013 inclui a atividade física como fator determinante e condicionante da saúde.

A inclusão permanente do código 2241-40, levou-se, em torno de 18 anos, para essa grande conquista da sociedade brasileira e principalmente para os profissionais de Educação Física, que agora poderão fazer parte e atuarem nos planos de saúde, 
abrindo um leque para a referia área e agregando valor para as demais áreas de saúde.

Assim sendo, o profissional de Educação Física, deverá e conseguirá desenvolver ações que propiciem a melhoria da qualidade de vida da população, a fim de reduzir os agravos e danos decorrentes das doenças não-transmissíveis, que favoreçam a redução do consumo de medicamentos e principalmente trabalhar em equipe multidisciplinar do SUS - Sistema Único de Saúde e conhecer a legislação específica da sua área de competência, para que não incorra em condutas e procedimentos que caracterizem práticas específicas de outras profissões da área da saúde. 


\section{REFERÊNCIAS}

BRASIL, Fundação Jorge Duprat Figueiredo de Segurança e Medicina do Trabalho. Disponível em: http://www.fundacentro.gov.br/estatisticas-de-acidentesde-trabalho/ciuo68-e-cbo77-revisitadas acesso em 03 de abril de 2020.

BRASIL, Ministério do Trabalho. CLASSIFICAÇÃO BRASILEIRA DE OCUPAÇÕES. Disponível em: http://www.mtecbo.gov.br/cbosite/pages/home.jsf, acesso em 03 de abril de 2020.

BRASIL, Ministério do Trabalho. PORTARIA № 397, DE 09 DE OUTUBRO DE 2002. Disponível em: https://www.camara.leg.br/proposicoesWeb/prop mostrarintegra. acesso em 03 de abril de 2020.

BRASIL, Presidência da República. Decreto no 1264 de 11 de outubro de 1994 Disponível em: http://www.planalto.gov.br/ccivil 03/decreto/1990-1994/D1264.htm acesso em 03 de abril de 2020.

BRASIL, Ministério da Saúde. Resolução CNS n. 218, de 6 de março de 1997. Reconhece as categorias profissionais consideradas como profissionais de saúde de nível superior. Diário Oficial da União. 5 maio 1997. Disponível em: http://sna.saude.gov.br/legisla/legisla/exerc_p/RES_CNS218_97exerc_p.doc, Acesso em: 15 de julho. 2019.

CONFEF. Lei 9696, de 1ำ de setembro de 1998. Dispõe sobre a Regulamentação da Profissão de Educação Física e cria os respectivos Conselho Federal e Conselhos Regionais de Educação Física. disponível em: https://www.confef.org.br/confef/conteudo. Acesso em: 10 de abril. 2020.

CONFEF. Profissional de Educação Física na Saúde esta na CBO disponível em: https://www.confef.org.br/confef/comunicacao/noticias. Acesso em: 10 de abril. 2020.

LAKATOS, Eva Maria. MARCONI, Marina de A. Fundamentos de Metodologia Científica. São Paulo: ebook, Atlas Editora, 2017.

NUNES, Everaldo Duarte. Saúde coletiva: história e paradigmas. Interface Comunic, Saúde, Educ. agosto 1998 